T b. Beling: Metamorphose der Zweiflügler-Gattung Sciara Meig.

\title{
Beitrag zur Metamorphose der Zweiflügler-Gattung Sciara Meig.
}

Von Theodor Beling, Forstmeister in Seesen am Harz.

(Fortsetzung.)

\section{Sciara dispar Bel.}

(Wien. Entom. Zeit. IV. pag. 307.)

Larve: $4-5 \mathrm{~mm}$ lang, $1.4 \mathrm{~mm}$ dick, gedrungen walzig, an den etwas verdünnten Leibesenden weiss, wasserhell, in der Mitte citronengelb, mit kleinem linsenförmigen schwarzbraunen glänzenden Kopfschilde, dessen Hinterrand in der Mitte einen kleinen ausgekerbten Vorsprung und zu dessen beiden Seiten flache Ausbuchtungen zeigt.

$\mathrm{Puppe:} \mathrm{blass} \mathrm{bräunlichgelb} \mathrm{mit} \mathrm{sehr} \mathrm{kleinen} \mathrm{braunen}$ punktförmigen Seitenstigmen, ठ $3 \mathrm{~mm}$ lang, $0.8 \mathrm{~mm}$ dick, Flügelscheiden bis Ende des zweiten, Fussscheiden bis Mitte des vierten Hinterleibssegmentes reichend; $O$ über doppelt so umfangreich $4 \mathrm{~mm}$ lang, $1.3 \mathrm{~mm}$ dick, Flügelscheiden bis Mitte, Fussscheiden bis Ende des dritten Hinterleibssegmentes reichend.

Eine Menge Larven fand ich am 13. Mai unter der Rinde eines alten in Vermoderung begriffenen Eichenstockes in einem Laubholzbestande. Dieselben sassen eng zusammengedrängt und mit einzelnen dünnen Fäden gespinnstartig überzogen. Die mitgenommenen Larven verpuppten sich am 16. und 17. Mai und am 24. dieses Monats erschienen viele Mücken, darunter aber nur ganz wenige $\hat{o}$.

\section{Sciara egregia Bel.}

Larve: im ausgewachsenen Zustande der Larve der Sc. rufiventris (siehe Nr. 10) in Grösse, Gestalt und Färbung so ähnlich, dass es mir nicht gelungen ist, charakteristische Unterschiede aufzufinden.

Etwa einhundert in ein dichtes Häufchen zusammengedrängte Larven wurden am 13. März in einem Buchenbestande mittleren Alters unter der Laubdecke des Bodens aufgefunden. Sie waren weiss bis auf den schwarzen Kopf, färbten sich aber, mit nach Hause genommen und in einem Gefässe auf einer Erdunterlage mit waldfeuchtem, in Verwesung begriffenen, öfter erneuerten Buchenstreulaube iiberdeckt und in einem nicht geheizten Zimmer unterhalten, schon nach Ablauf der ersten Woche lebhaft citronengelb. 
Am 1. Mai hatten sie sich in der Weise verpuppt, dass jede Puppe mit einer erdigen Hülle, ähnlich wie die Puppen von Sc. rufiventris es zu sein pflegen, umgeben war. Vom 9. Mai an erschienen zahlreich die Imagines, erst $\hat{\sigma}$, dann viel mehr $\bigcirc$. Am 21. März eines anderen Jahres wurden in humoser Erde unter einem Strauche von Sambucus nigra L. inmitten einer Wiesenhecke zwei Larven gefunden, aus deren einer zu Hause am 29. Mai ein $q$ hervorging (I, Jahrg. 1874, pag. 4).

\section{Sciara glabricollis Winn.}

Larve: $4-5 \mathrm{~mm}$ lang, $0.6 \mathrm{~mm}$ dick, ziemlich scharf gegliedert, an den ersten drei Leibessegmenten weiss glasig, sonst citronengelb, mit mässig grossem schwärzlichbraunen, in der Mitte helleren Kopfe.

Puppe: bis $3.5 \mathrm{~mm}$ lang, $0.8 \mathrm{~mm}$ dick, bis auf die klar weissen, später sich schwärzenden und dann stark firnissartig glänzenden Flügel-, Fuss- und Fühlerscheiden lebhaft citronengelb, an jeder Leibesseite mit einer Längenreihe punktförmiger schwärzlicher, leicht bemerkbarer Stigmen. Flügelscheiden bis Ende des zweiten, Fussscheiden bis Anfang oder Mitte des vierten Hinterleibssegmentes reichend.

Am 16. November wurde ein zusammengeballter haselnussgrosser Klumpen Larven unter der Rinde des Stockes einer im Jahre zuvor gefällten Kiefer (Pinus sylvestris L.) im Walde, etwa zur Hälfte in der zernagten Rinde steckend, gefunden. Die in einem nicht geheizten Zimmer unterhaltenen Larven blieben bis Anfang März des folgenden Jahres gehäuft beisammen, frassen sich nach dem 20. des letzgenannten Monats tiefer in die feucht erhaltene Rinde hinein und verpuppten sich daselbst in den letzten Tagen des Monats April ohne Anfertigung eines Gespinnstes. Nach 16tägiger Puppenruhe begannen die Imagines zu erscheinen. In einem späteren Jahre wurden am 20. Mai unter der Rinde alter Kiefernstöcke im Walde theils Larven, theils Puppen, letztere in überwiegender Zahl gefunden. Aus mitgenommenen Larven gingen bis zum 21. Mai Puppen und aus diesen schon nach sechstägiger Ruhe Imagines hervor. (I, Jahrg. 1871, pag. 851.)

\section{Sciara hortulana Bel.}

La rve: $3-4 \mathrm{~mm}$ lang, $0.5 \mathrm{~mm}$ dick, orangegelb mit Ausschluss der weissen vorderen zwei und letzten beiden Leibes- 
segmente und mit intensiv braun durchscheinendem Darminhalte. Der etwa ein Drittheil der grössten Körperbreite messende Kopfschild hell bräunlich gelb, vorn und am Hinterrande schmal schwarzbraun gesäumt.

P u p pe: 2.5 bis $3 \mathrm{~mm}$ lang, $0.5-0.7 \mathrm{~mm}$ dick, orangegelb wie die Larve, beim ô die Flügelscheiden bis Ende des zweiten, die Fussscheiden bis Ende des dritten Hinterleibssegmentes, beim $O$ die Fussscheiden bis Mitte des dritten Hinterleibssegmentes reichend.

Larven und vereinzelte Puppen wurden in theilweise oder ganz abgestorbenen Apfelbäumen eines Gartens zwischen Holz und Rinde, zum Theil in letzterer innerhalb der Bastschicht sitzend, am 2. April gefunden. Aus am 7. dieses Monats eingezwingerten Puppen gingen schon am folgenden Tage zahlreiche Mücken hervor. Die Puppen lagen zum Theil frei, zum Theil jede für sich in einer schmutzig bräunlichgelben, etwas durchscheinenden, eng anschliessenden, häutigen Umhüllung unter oder innerhalb der Rinde (I, Jahrg. 1874, pag. 9).

\section{Sciara nigrescens Winn.}

Larve: bis $5 \mathrm{~mm}$ lang, $1.5 \mathrm{~mm}$ dick, ausgestreckt nach vorn hin stark spindelig verdünnt, am Hinterrande stumpf abgerundet, anfänglich weiss, später orangegelb mit weissen wasserhellen Leibesenden, schwarzbraun durchscheinendem Darminhalte und kleinem schwarzen, glänzenden Kopfe.

P uppe: $3-4 \mathrm{~mm}$ lang, $0.8-1.4 \mathrm{~mm}$ dick, orangegelb, an jeder Leibesseite mit einer Längenreihe heller runder Flecke inmitten deren die kleinen punktförmigen Stigmen befindlich. Fussscheiden bei $\sigma$ bis zum Ende des siebenten, bei $ᄋ$ bis zum Anfange des sechsten Hinterleibssegmentes reichend.

Die Mücke ist in den Laubholzwaldungen der hiesigen Gegend in manchen Jahren ausserordentlich häufig. Die von der in Verwesung begriffenen unteren Schicht der Laubdecke des Bodens sich nährenden Larven findet man haufenweise dicht zusammengedrängt mitunter in solchen Massen, dass auf grossen Flächen die Laubdecke des Bodens, zumal wenn solche aus Hainbuchenblättern (Carpinus Betulus L.) besteht, fast ganz von ihnen aufgezehrt wird. Die Mücke erscheint im Monat Mai. Besonders bemerkenswerth ist, dass die Larven dieser Sciara 
aus ihren Excrementen und aus ganz fein zerbissenen Streulaubtheilen feste Gespinnste zu ihrer Verwandelung anfertigen. Diese Gespinnste liegen unter der Laubdecke des Bodens, ragen zuweilen mit ihrem unteren Theile etwas in die Erde hinein, sind unregelmässig quadratisch oder rundlich, mehrentheils etwas mehr in die Länge als in die Breite gedehnt, $2-4 \mathrm{~cm}$ lang, 2 auch wohl bis $3 \mathrm{~cm}$ breit, $6-8 \mathrm{~mm}$ dick, gleichen im äusseren Ansehen mitunter einigermassen den von Regenwürmern auf dem Boden zurückgelassenen Excrementenhäufchen und machen sich, obschon theils weniger, theils mebr mit Laubblättern bedeckt, dem darauf erst einmal eingeübten Auge leicht bemerklich. Die Larven stecken darin mit der Längenachse ihres Leibes vertical meist mit den Köpfen nach oben gerichtet in gesonderten häutigen, festen, ganz verschlossenen Hüllen mehr oder weniger eng aneinander gedrängt, von aussen nicht sichtbar. Nach dem Ausschlüpfen der Mücken haben die Gespinnste das Ansehen von zierlichen Waben oder Wachsscheiben eines Bienenstockes und sie sind so fest, dass man sie mitunter noch im Herbste ganz unversehrt unter der Laubdecke des Bodens findet (II, Jahrg. 1871, pag. 81).

\section{Sciara tremulae Bel.}

Larve: $4-5 \mathrm{~mm}$ lang, $0.6 \mathrm{~mm}$ dick, citronengelb, mit weissen Leibesenden, braun durchscheinendem Darminhalte und kleinem schwarzen, glänzenden Kopfschilde.

$\mathrm{Puppe:}$ bis $3.5 \mathrm{~mm}$ lang, $0.7 \mathrm{~mm}$ dick, orangegelb, die Flügelscheiden bis Ende des dritten, die Fussscheiden bis nahe zum Ende des fünften Hinterleibssegmentes reichend.

Die Larven fand ich wiederholt in Häufchen bis zu 10 Stück dicht zusammengedrängt in den von Saperda populnea L. angelegten, aber unlängst verlassenen Gängen jüngerer Stämme und Zweige der Aspe (Populus tremula L.), wo sie sich von den von den Saperda-Larven zurückgelassenen Excrementen und Nagespänen nährten. Die Verpuppung geschah im Monate Mai und nach etwa siebentägiger Ruhe gingen aus den Puppen die Imagines hervor (I, Jahrg. 1874, pag. 7). 


\section{$2 \mathrm{BHL}$ Biodiversity Heritage Library}

Beling, Theodor. 1886. "Beitrag zur Metamorphose der Zweiflügler-Gattung Sciara MEIG." Wiener entomologische Zeitung 5, 71-74.

https://doi.org/10.5962/bhl.part.20558.

View This Item Online: https://www.biodiversitylibrary.org/item/43771

DOI: https://doi.org/10.5962/bhl.part.20558

Permalink: https://www.biodiversitylibrary.org/partpdf/20558

\section{Holding Institution}

Smithsonian Libraries

\section{Sponsored by}

Smithsonian

\section{Copyright \& Reuse}

Copyright Status: NOT_IN_COPYRIGHT

This document was created from content at the Biodiversity Heritage Library, the world's largest open access digital library for biodiversity literature and archives. Visit BHL at https://www.biodiversitylibrary.org. 